\title{
Eficacia del programa educativo "por un útero feliz" para mejorar conocimientos y actitudes hacia la vacuna del virus del papiloma humano en estudiantes del quinto grado de primaria de la institución Educativa Las Mercedes, Juliaca
}

\author{
Efficacy of the educational program "for a happy uterus" to improve knowledge and \\ attitudes towards the vaccine of human papilloma virus in students of the fifth grade \\ of primary school of the Institución Educativa Las Mercedes, Juliaca
}

Yessica Huanca Yanapa ${ }^{1 *}$, Julia Huisa Machaca², Ruth Yenny Chura Muñuico

\section{RESUMEN}

Objetivos: Determinar la eficacia del programa educativo "Por un útero feliz" para mejorar conocimientos, actitudes hacia la Vacuna del virus del Papiloma Humano en estudiantes del quinto grado de primaria de la Institución Educativa "las Mercedes" en comparación a un grupo control. Materiales y Métodos: fue de tipo cualitativo con diseño de investigación cuasi experimental; en una muestra de 60 escolares mujeres, para recolectar los datos se utilizó la técnica de la encuesta en los que se aplicaron un pre test y post test, tanto al grupo control y experimental. Resultados: en el pre test son: un $90.6 \%$ presentó un nivel bajo, seguido de un $9.4 \%$ presentó un nivel medio y un $0 \%$ con un nivel alto y en actitud presentaron: un $59.4 \%$ rechazo, seguido de un $34.4 \%$ presentó indiferencia y un $6.3 \%$ presentó aceptación; luego de la intervención en la etapa post prueba se obtuvieron los siguientes resultados: un $96.9 \%$ presentó un nivel alto, seguido de un $3.1 \%$ presentando un nivel medio y un $0 \%$ de los escolares presentó un nivel bajo y en actitud un $93.8 \%$ evidenció aceptación, seguido de un $6.3 \%$ mostraron indiferencia y un $0 \%$ muestra rechazo. Aplicando la prueba T para muestras relacionadas se acepta la hipótesis alterna. Conclusiones: el programa educativo "por un útero feliz" es eficaz para mejorar los conocimientos y actitudes frente a la vacuna del VPH en escolares.

Palabras claves: Cáncer de cuello uterino, vacuna VPH, actitudes, programa educativo. (Fuente: DeCS BIREME)

\section{ABSTRACT}

Objetives: determine the effectiveness of the educational program "for a happy uterus" to improve knowledge, attitudes towards Vaccine Human Papilloma students in the fifth grade of School "Mercedes" compared to a control group. Materials and Methods: the methodology was qualitative with quasi-experimental research design; in a sample of 60 school women, to collect the data the survey technique in which a pre-test and post-test, both the control and experimental group were applied was used. Results: in the pretest are: $90.6 \%$ had a low level, followed by a $9.4 \%$ showed a medium level and $0 \%$ to a high standard and attitude showed: $59.4 \%$ rejection, followed by a $34.4 \%$ presented indifference and $6.3 \%$ presented acceptance; after the intervention in the post-test stage, the following results were obtained: A $96.9 \%$ had a high level, followed by a $3.1 \%$ having an average level and $0 \%$ of schoolchildren presented a low and attitude level $93.8 \%$ showed acceptance, followed by a $6.3 \%$ showed indifference and rejection shows $0 \%$. Applying the $\mathrm{T}$ test for related samples the alternative hypothesis is accepted. Conclusions: that the educational program "for a happy uterus" is effective in improving knowledge and attitudes towards HPV vaccine in school.

Keywords: Cervical HPV vaccine, attitudes, cancer education program. (Source: MeSH NLM)

${ }^{1}$ Enfermera SERUMS, Puesto de Salud Santa Clara, Cajamarca, Perú

'2Licenciado en Enfermería, Universidad Peruana Unión, Lima, Perú

${ }^{3}$ Docente de Escuela Profesional de Enfermería, Universidad Peruana Unión, Lima, Perú. 
Eficacia del programa educativo "por un útero feliz" para mejorar conocimientos y actitudes hacia la vacuna del virus del papiloma humano en estudiantes del quinto grado de primaria de la institución Educativa Las Mercedes, Juliaca

\section{INTRODUCCIÓN}

Al pasar de los años el uso de nuevas vacunas ha despertado inquietud y desconfianza en la población, tanto a nivel nacional e internacional se evidencia esta problemática en las campañas de vacunación, debido a las bajas coberturas de esta vacuna; así mismo la Organización Mundial de la Salud (2013) menciona que cada año, aproximadamente 9 millones de personas en el mundo padecen cáncer y 5 millones mueren por su causa. Se estima que, actualmente existen unos 14 millones de personas enfermas con cáncer y las consecuencias económicas del mismo lo convierten en un problema de salud importante para la humanidad. Más del $90 \%$ de las muertes se concentran en los países de ingresos bajos y medios, donde el acceso a los oportunos servicios de detección y tratamiento es muy limitado. Laura (2011) menciona que, en Latinoamérica, el cáncer de cuello uterino se ubica entre las primeras causas de muerte por cáncer en la mujer, superando al cáncer de mama, siendo los tipos de cáncer que presentan la mayor tasa de mortalidad o incidencia. Y Perú no está ajeno a esta realidad estudios recientes muestran que en la actualidad el cáncer de cuello uterino, representa la segunda causa de muerte en nuestro país. Asimismo, en el 2011 se produjeron más de 4 mil muertes por esta enfermedad, lo que equivale a la muerte de una mujer peruana cada 3.5 horas por cáncer de cuello uterino. Toda la evidencia científica actual muestra que la causa principal del cáncer de cuello uterino es el Virus Papiloma Humano, según (MINSA, 2015).

La Organización Mundial de la Salud (2013), recomienda incluir la vacunación contra el VPH en los programas Nacionales de Inmunizaciones donde la prevención del cáncer de cuello uterino sea una prioridad de salud pública. Como también la Organización Panamericana de la Salud (2013) menciona que el uso masivo de las vacunas ha sido posible salvar a miles de personas, asimismo la vacuna tiene una eficacia mayor al $90 \%$ con el esquema de las 3 dosis y hay evidencias de que previene la infección por los principales agentes oncológicos.

EI MINSA (2015) menciona que la vacunación contra el VPH es una intervención clave para la disminución de la morbi-mortalidad por cáncer de cuello uterino por ello recomienda la administración temprana a las edades de 9 13 años antes del inicio de la actividad sexual, cumpliendo las 3 dosis según esquema de vacunación.

El personal de enfermería juega un papel importante en la promoción de la salud y prevención de enfermedades. El presente trabajo de investigación surgió durante el internado comunitario donde se evidenció que las escolares presentaban un desconocimiento, incertidumbre y miedo hacia la vacuna VPH. Siendo por tanto fundamental la participación de los escolares es necesario saber cuánto conoce de un determinado tema que beneficie su salud. Por lo tanto, existe un interés de investigar el nivel de conocimiento y la actitud de los escolares del quinto año de la Institución Educativa las Mercedes hacia a la vacuna del VPH, debido a que el cáncer de cuello uterino en la región Puno es la primera causa de muerte en mujeres.

\section{MATERIAL Y MÉTODOS}

El presente trabajo de investigación corresponde a un estudio tipo cualitativo, de nivel de aplicativo, siendo el abordaje metodológico cuasi experimental se cuenta con dos grupos una de control y el otro experimental; en este diseño se aplica al grupo control un pre-test (O1), finalmente el post-test (O2) a diferencia del grupo experimental se aplica un pre-test (O1), después el tratamiento $(X)$ y finalmente el post-test $(\mathrm{O} 2)$. El resultado es la valoración del cambio ocurrido entre el pre y pos-test del grupo experimental en comparación al grupo control.

\section{Población y muestra}

La población finita estuvo conformada por 120 adolescentes entre varones y mujeres del quinto grado de primaria de la institución educativa las mercedes, considerando solo a las mujeres las cuales conforma la suma de 60 estudiantes. Se trabajó como grupo control (G.C.) con las secciones "A" y "B" y como Grupo experimental (G.E.) con las secciones "C" $y$ " $D$ ". Según Hernández et al. (2010) menciona que la muestra es no probabilística, intencional o por conveniencia, ya que se hace una elección de los elementos no dependen de la probabilidad sino de causas relacionadas con las características de la investigación o quien hace la muestra.

\section{Instrumento}

El instrumento utilizado fue elaborado por Tafur (2012) que mide conocimientos y actitudes hacia 
la vacuna contra el VPH en los escolares; este cuestionario fue sometidos a juicio de jueces expertos y a prueba piloto para su validez y confiabilidad; teniendo una confiabilidad del 0.67 .

Para la intervención se aplicó el programa educativo "por un útero feliz" que consta de cuatro módulos dentro de ellos se trabajó de tres a dos sesiones por módulo que hicieron un total de nueve sesiones desarrolladas cuatro veces por semana, durante dos meses.

\section{Proceso y análisis de datos}

En cuanto al procesamiento estadístico; se utilizó la prueba T es una prueba estadística para evaluar si dos grupos difieren entre sí de manera significativa respecto a sus medias en una variable. La prueba t se basa en una distribución muestral o poblacional de diferencia de medias conocida como la distribución $t$ de Student que se identifica por los grados de libertad, los cuales constituyen el número de maneras en que los datos pueden variar libremente. Del mismo modo para la prueba de hipótesis se utilizó la prueba de comparación de medias para muestras relacionadas e independientes en donde los resultados de una pre-prueba con los de un pos prueba comparan las medias y la varianza de un grupo en dos momentos diferentes (Hernández, et al., 2010).

\section{RESULTADOS}

Según la tabla 1 se puede observar que el nivel de conocimiento general sobre el VPH obtenidos de los escolares de la I.E. las Mercedes del grupo experimental antes de la aplicación del programa educativo "por un útero feliz", el $90,6 \%$ de las escolares presentan un nivel de conocimiento bajo y solo un $9,4 \%$ de ellas tiene un conocimiento medio; a su vez se puede observar que después de la aplicación del programa educativo "por un útero feliz, estos porcentajes han variado; el 96,6\% presentan un conocimiento alto y solo un $3,1 \%$ de ellas presentan un conocimiento medio, lo que evidencia que el programa educativo es eficaz para mejorar los conocimientos hacia la Vacuna del Papiloma Humano en las escolares del quinto grado de primaria.

Tabla 1

Nivel de conocimientos hacia la vacuna del Virus del Papiloma Humano en las escolares del quinto grado de primaria de la I.E. las Mercedes del grupo control y experimental antes y después de la intervención del Programa Educativo.

\begin{tabular}{|c|c|c|c|c|c|c|}
\hline & & & \multicolumn{2}{|c|}{ G. Control } & \multicolumn{2}{|c|}{ G. Experimental } \\
\hline & & & Pre & Post & Pre & Post \\
\hline \multirow{6}{*}{ Conocimientos generales } & \multirow{2}{*}{ Bajo } & $\mathrm{N}$ & 20 & 24 & 29 & 0 \\
\hline & & $\%$ & 71,4 & 85,7 & 90,6 & 0,0 \\
\hline & \multirow{2}{*}{ Medio } & $\mathrm{N}$ & 8 & 4 & 3 & 1 \\
\hline & & $\%$ & 28,6 & 14,3 & 9,4 & 3,1 \\
\hline & \multirow{2}{*}{ Alto } & $\mathrm{N}$ & 0 & 0 & 0 & 31 \\
\hline & & $\%$ & 0,0 & 0,0 & 0,0 & 96,9 \\
\hline \multirow[t]{2}{*}{ Total } & & $\mathrm{N}$ & 28 & 28 & 32 & 32 \\
\hline & & $\%$ & 100,0 & 100,0 & 100,0 & 100,0 \\
\hline
\end{tabular}

${ }^{*}$ sig $=0.00$

En la tabla 2 se observa en el grupo experimental en la etapa pre- prueba de la aplicación del programa educativo "por un útero feliz", los resultados fueron los siguiente: el 59,4\% presentaba un nivel de rechazo, seguido del $34,4 \%$ con niveles de Indiferencia y $6,3 \%$ presenta un nivel de aceptación; después de la aplicación de programa eductaivo "por un útero feliz" se aprecia que la mayoría de las escolares presentan una actitud de aceptación con un porcentaje de 93,8\%, seguido de una indiferencia de $6,3 \%$ y un por ultimo un $0 \%$ de rechazo, lo que evidencia que el programa educativo es eficaz para la mejora de las actitudes ante la vacuna VPH en las escolares. 
Eficacia del programa educativo "por un útero feliz" para mejorar conocimientos y actitudes hacia la vacuna del virus del papiloma humano en estudiantes del quinto grado de primaria de la institución Educativa Las Mercedes, Juliaca

Tabla 2

Nivel de actitud general hacia la vacuna del VPH en escolares del quinto grado de primaria de la I.E. las Mercedes del grupo control y experimental antes y después de la intervención del Programa Educativo.

\begin{tabular}{|c|c|c|c|c|c|c|}
\hline & & & \multicolumn{2}{|c|}{ Grupo Control } & \multicolumn{2}{|c|}{ Grupo Experimenta } \\
\hline & & & Pre & Post & Pre & Post \\
\hline \multirow{4}{*}{ Actitud } & Rechazo & $\begin{array}{l}\mathrm{N} \\
\%\end{array}$ & $\begin{array}{l}12 \\
42,9\end{array}$ & $\begin{array}{l}16 \\
57,1\end{array}$ & $\begin{array}{l}19 \\
59,4\end{array}$ & $\begin{array}{l}0 \\
0,0\end{array}$ \\
\hline & Indiferencia & $\begin{array}{l}\mathrm{N} \\
\%\end{array}$ & $\begin{array}{l}16 \\
57,1\end{array}$ & $\begin{array}{l}12 \\
42,9\end{array}$ & $\begin{array}{l}11 \\
34,4\end{array}$ & $\begin{array}{l}2 \\
6,3\end{array}$ \\
\hline & Aceptación & $\begin{array}{l}\mathrm{N} \\
\%\end{array}$ & $\begin{array}{l}0 \\
0,0\end{array}$ & $\begin{array}{l}0 \\
0,0\end{array}$ & $\begin{array}{l}2 \\
6,3\end{array}$ & $\begin{array}{l}30 \\
93,8\end{array}$ \\
\hline & Total & $\begin{array}{l}N \\
\%\end{array}$ & $\begin{array}{l}28 \\
100,0\end{array}$ & $\begin{array}{l}28 \\
100,0\end{array}$ & $\begin{array}{l}32 \\
100,0\end{array}$ & $\begin{array}{l}32 \\
100,0\end{array}$ \\
\hline
\end{tabular}

${ }^{*}$ sig $=0.00$

\section{DISCUSIÓN}

Mediante la presente investigación fue posible evaluar el nivel de conocimiento y actitud acerca de la vacuna contra el virus del papiloma humano en mujeres adolescentes del quinto año de primaria; respecto al objetivo general los resultados muestran que antes de la aplicación del programa "por un útero feliz", un $90.6 \%$ presentó un nivel bajo, $9.4 \%$ y un nivel medio y un $0 \%$ un nivel alto y en actitud en el pre test presentaron un $59.4 \%$ de rechazo, un $34.4 \%$ presentó una indiferencia y un 6.3\% muestra aceptación. Encontrándose un mayor porcentaje de las adolescentes con un nivel bajo de conocimiento y una mayor actitud de rechazo, siendo que esto podría ocasionar rechazo hacia la vacuna contra el VPH; luego de la aplicación del programa "por un útero feliz" se obtuvieron los siguientes resultados, un $96.9 \%$ presentó un nivel alto, $3.1 \%$ presentó un nivel medio y un $0 \%$ de los escolares presentaron un nivel bajo y en la variable actitud un $93.8 \%$ presentaron una actitud de aceptación, seguido de un $6.3 \%$ presentaron un nivel de indiferencia y un $0 \%$ presentaron un rechazo. Con lo que concluimos que nuestro programa denominado "por un útero feliz" es eficaz para poder mejorar conocimientos y actitud en las mujeres escolares del quinto año de primaria que es el grupo con el cual trabajamos, conformado por 32 alumnas de las secciones "C" y " $D$ " de la Institución Educativa "Las Mercedes" de la ciudad de Juliaca. Este trabajo guarda relación con la investigación presentado por Gutman y Traviesa (2013) realizaron el trabajo de investigación titulado "Nivel de conocimiento y aceptabilidad de la vacuna contra el Virus del Papiloma Humano (HPV) en estudiantes secundarios de la ciudad de Presidencia Roque Sáenz Peña, Chaco" en Argentina cuyo objetivo fue relacionar la aceptabilidad de la vacuna contra el (HPV) y el conocimiento previo sobre éste y su relación al Cáncer Cérvico- Uterino, con el diseño metodológico de este estudio es de tipo descriptivo de corte transversal, en el que se analizó el nivel de conocimiento sobre el HPV, su prevención y la posible relación entre estas variables y la aceptabilidad de la vacuna, el conocimiento que los estudiantes obtuvieron según la clasificación utilizada fue "nivel bajo". Si se comparan las instituciones entre sí se puede deducir que, a mayor conocimiento, más probabilidad de que las personas que tuviesen la oportunidad y se les recomienda se colocasen la vacuna contra el HPV; llegaron a la conclusión que el conocimiento general que tienen los estudiantes acerca del HPV resulta insuficiente para garantizar una adecuada prevención, pero, aun así, un $78 \%$ de los mismos estaría dispuesto a recibir la vacuna, en caso de tener la oportunidad de hacerlo. Asimismo, el trabajo guarda relación con la investigación presentado por Tafur (2012), tiene un enfoque cuantitativo, método no experimental diseño descriptivo, de corte trasversal. La muestra fue constituida por los estudiantes de dos escuelas mixtas: una estatal de nivel primario y otra privada con una población aproximada de 1800 estudiantes; presentando como resultado el nivel de conocimiento en las preadolescentes frente a la vacuna contra el virus del papiloma 
humano (VPH) fue medio (60\%), con tendencia semejante en el análisis por dimensiones generalidades del VPH y conocimientos sobre la vacuna, destacándose el desconocimiento de forma de transmisión y síntomas.

En la actitud, el $60 \%$ se clasifico en indiferente, al igual que por dimensiones: cognitiva $(68 \%)$, afectivo $(69 \%)$ y conductual $(70 \%)$. Llegando a la conclusión que el nivel de conocimiento sobre la vacuna contra el VPH fue medio con una actitud de indiferencia frente a la vacuna. Es necesario fomentar mayor conciencia sobre la importancia de la vacuna contra el virus del papiloma humano en la población.

Declaración de financiamiento y de conflictos de interés:

El estudio fue financiado por los autores, quienes declaran no tener conflictos de interés.

\section{Correspondencia}

Yessica Huanca Yanapa. Dirección: Puesto de Salud Santa Clara, Cajamarca, Perú.

Correo electrónico: yesha_dan@upeu.edu.pe

\section{REFERENCIAS BIBLIOGRÁFICAS}

Dirección General de epidemiologia. (Noviembre de 2013). Obtenido de Analisis de situacion de cancer en el Peru: http://www.dge.gob.pe/portal/ docs/asis_cancer.pdf.

Gutman, M. F., Luna, M. C., \& Traviesa, L. M. (2013). nivel de conocimientos y aceptabilidad de la vacuna contra el virus del ppiloma humano (VPH) en estudios secundarios de la ciudad de presidencia Roque Sanz. Revista de la facultad de medicina, 1-5.

Hernández, R., Fernandez, C., \& Batista, M. d. (2010). Metodología de la investigación (Vol. Quinta edicion). México: Interamericana editores,S.A. DE C.V.

Organización Mundial de La Salud. (marzo de 2013). Papilomavirus humanos (PVH) y cáncer cervicouterino. Recuperado el 24 de febrero de 2016, de centro de prensa Papilomavirus humanos $(\mathrm{PVH})$ y cáncer cervicouterino: http:// www.who.int/mediacentre/factsheets/fs380/es/

Organización Panamericana de la Salud. (19 de marzo de 2013). Organización Panamericana de la Salud. Recuperado el 24 de febrero de 2015, de POSICIÓN DE la representación de la organización panamericana de la salud: http:// www.paho.org/par/index.php?option=com_co ntent\&view=article\&id=914: posicion-opssobre-vacuna-contra-virus-papiloma-humanovph\&Itemid=258

Laura C, A. (11 de febrero de 2011 ). Perfil.com.salud. Recuperado el 25 de febrero de 2016, de El VPH afecta al $50 \%$ de la población sexualmente

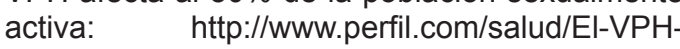
afecta-al-50-de-la-poblacion-sexualmenteactiva-20110211-0010.html.

Ministerio de salud. (17 de marzo de 2015). Ministerio de salud. Recuperado el 29 de Febrero de 2016, de Plan de Salud Escolar: Más de 475 mil niñas serán vacunadas contra el Virus de Papiloma Humano: http://www.minsa.gob. pe/?op $=51 \&$ nota $=16252$

MINSA. (03 de Marzo de 2015). MINSA. Obtenido de Plan Esperanza reconocido por National Cancer Institute y OPS como modelo de política de salud: http://www.minsa.gob.pe/?op=51\&nota=16185

MINSA. (17 de marzo de 2015). Sala de prensa plan de salud escolar. Recuperado el 22 de Febrero de 2016, de Plan de Salud Escolar: Más de 475 mil niñas serán vacunadas contra el Virus de Papiloma Humano: http://www.minsa.gob. $\mathrm{pe} / \mathrm{?op}=51 \&$ nota $=16252$

Organizacicon Mundial de la Salud. (septiembre de 2013). Organizacicon Mundial de la Salud. Recuperado el 29 de febreo de 2016, de Salud de la Mujer: http://www.who.int/mediacentre/ factsheets/fs334/es/

Organización mundial de la salud. (marzo de 2015). Documento de posición de la OMS sobre las vacunas contra el virus del papiloma humano(VPH). Recuperado el 15 de febrero de 2016, de Organizacion mundial de la salud: http:// www.who.int/immunization/documents/HPV_PP_ introd_letter_Spanish.pdf

Organizacion panamericana de la salud. (19 de Marzo de 2013). Posición de la representación de la organización panamericana de la salud. Recuperado el 15 de febrero de 2016, de OPS: http://www.paho.org/par/index.php?option=com content\&view=article\&id=914: posicion-opssobre-vacuna-contra-virus-papiloma-humanovph\&Itemid=258

Tafur, C. M. (2012). Conocimientos y actitudes frente a la vacuna contra el virus del papiloma humano en adolescentes de $5^{\circ}$ año de primaria. Lima. Recuperado el 16 de 02 de 2016

Recibido: 25/04/17

Aceptado: 05/10/17 